\title{
Avaliação postural por fotometria em pacientes hemiplégicos
}

\section{Postural assessment by photometry in hemiplegic patients}

\author{
Barbara Caetano Pereira'; Carla Christina Medalha² \\ ${ }^{1}$ Acadêmica do curso de Fisioterapia - Unifesp, Campus da Baixada Santista. \\ ${ }_{2}^{2}$ Doutora em Ciências Fisiológicas - UFSCar. Docente do curso de Fisioterapia - Unifesp, Campus da Baixada Santista.
}

ENDEREÇO PARA CORRESPONDÊNCIA

Santos - SP [Brasil]

barbaracaetano@gradunifesp.br

\begin{abstract}
RESUMO
A avaliação postural é de fundamental importância para o planejamento de um tratamento fisioterapêutico. No entanto, é escassa a literatura referente às alterações posturais de pacientes que sofreram um acidente vascular encefálico (AVE); em razão disso, o objetivo deste estudo foi comparar a postura estática de pacientes hemiplégicos à de indivíduos sem lesões neurológicas. A avaliação consistiu no registro fotográfico das vistas anterior, lateral e posterior. As imagens foram analisadas pelo Software de Avaliação Postural (SAPO). A análise estatística pelo Teste de Soma de Postos de Mann-Whitney confirmou que, no grupo acometido, os valores de ângulos são maiores quando comparados aos do grupo controle, nas vistas anterior e posterior. Concluiu-se que pacientes acometidos por um AVE apresentam assimetrias posturais significativas que devem ser minimizadas por meio do tratamento fisioterapêutico adequado.
\end{abstract}

Descritores: Acidente vascular encefálico; Fisioterapia; Postura.

\begin{abstract}
The postural assessment is fundamental to elaboration of a physiotherapeutic treatment program. However, rare literature referring to postural disorders in stroke patients is found. The aim of this study was to compare the posture of hemiplegic patients with control individuals without any neurological injury. The assessment consisted of photographic register of patients in anterior, lateral and posterior sights. The images had been analyzed through the Software of Postural Evaluation (SAPO). The statistics analyses were performed by the Mann-Whitney Rank Sum Test, and it was verified that affected group presented increased values of angles when compared to group control, in anterior and posterior. It can be concluded that stroke has consequences of postural asymmetries, which could be minimized by adjustments in physiotherapeutic approach.
\end{abstract}

Key words: Hemiplegic; Physical therapy; Posture. Stroke. 


\section{INTRODUÇÃO}

Postura pode ser definida como "[...] uma posição ou atitude do corpo, o arranjo relativo das partes do corpo para uma atividade específica, ou uma maneira característica de alguém sustentar seu corpo"1. ${ }^{\prime \prime}$ O termo postura, segundo Shumway-Cook e Woolacott ${ }^{2}$, também é usado para o alinhamento biomecânico do corpo e sua orientação em relação ao ambiente. $\mathrm{Na}$ postura adequada, a musculatura, as articulações e a estrutura esquelética estão em estado de equilíbrio ${ }^{3,4}$, além de proteger as estruturas corporais contra lesões ou deformidades ${ }^{5,}{ }^{6}$.

A definição de alinhamento postural, proposta por Kendal' ${ }^{7}$ é uma referência utilizada internacionalmente como padrão, e a fisioterapia considera como alteração postural qualquer assimetria entre os segmentos corporais. A avaliação é sistematicamente feita de modo qualitativo. No entanto, a simetria não é regra e sim exceção, quando se trata de postura e de avaliação qualitativa, indiscutivelmente nos referimos a uma de com menor credibilidade que uma medida quantitativa ${ }^{6,8}$. A importância da avaliação do alinhamento postural justifica-se pelo conceito de que o estresse mecânico tem repercussões clínicas, gera conseqüências no tecido conjuntivo, nos músculos e nas articulações, contribuindo para a degeneração articular e as tensões musculares inadequadas ${ }^{9}$.

A musculatura do corpo humano apresenta um determinado "nível de tensão" geral, necessário para manutenção da postura corporal contra a gravidade, que é definido como tônus postural ou antigravitacional ${ }^{3,4}$. Essa capacidade de manter a estabilidade na postura ereta é um processo de aprendizagem do sistema nervoso central (SNC), e a aquisição e o refinamento do controle postural envolvem a identificação de um relacionamento coerente e estável entre as informações sensoriais e as ações motoras necessárias para manutenção de uma posição corporal desejada ${ }^{10}$. O SNC "aprende" com a utilização de informações dos elementos biomecânicos passivos, do sistema sensorial e dos músculos, interpretando e organizando as informações sensoriais de várias estruturas, e apresenta respostas com base em experiências anteriores ${ }^{11}$. As lesões no SNC estão muitas vezes associadas à manutenção de posturas características, como na Doença de Parkinson ou na Hemiplegia, relacionadas à perda de força muscular, redução da coordenação e do equilíbrio, e às mudanças de tônus muscular². As alterações tônicas são comuns após lesões neurológicas, pois essa regulação da função muscular é controlada por estruturas do $\mathrm{SNC}^{12}$. As disfunções tônicas são comprometimentos primários após lesões neurológicas e podem variar desde o aumento tônico (hipertonia, ou seja, espasticidade ou rigidez) até sua redução (hipotonia, flacidez). Se não tratado adequadamente, o tônus anormal pode levar ao desenvolvimento de comprometimentos secundários como contraturas, assimetrias posturais e deformidades ${ }^{2,13}$. Com isso é bom inferir que um bom equilíbrio tônico seja necessário para uma simetria postural ${ }^{4}$. Desequilíbrios tônicos associados com outros comprometimentos neurológicos primários, como a fraqueza muscular e a incoordenação, estão clinicamente vinculados à postura dos pacientes neurológicos, como a típica de um hemiplégico (com inclinação lateral de todo o corpo e redução da descarga de peso entre os membros) ou de um parkinsoniano (postura em flexão de tronco, com rotação interna de quadril e gleno-umeral e base alargada) ${ }^{13}$.

A avaliação postural é de fundamental importância para planejar o tratamento fisioterapêutico e acompanhar a evolução e os resultados ${ }^{7,14}$. No entanto, há poucos registros na literatura médica sobre as alterações posturais, que se processam a longo prazo de pacientes que sofreram lesões neurológicas, especialmente acidente vascular encefálico (AVE). O AVE é a terceira causa de morte no mundo, depois das cardiopatias em geral e do câncer. Cerca de 40 a 50\% dos indivíduos que sofrem um AVE vão a óbito após seis meses, e a maioria dos sobreviventes exibirá deficiências 
neurológicas e incapacidades residuais significativas, segundo a Organização Mundial de Saúde $^{15}$. As conseqüências do AVE nos indivíduos são diversas e, geralmente, permanecem por longos períodos, podendo atingir os três níveis, propostos pela OMS no modelo de Classificação Internacional de Funcionalidade (CIF): o de estrutura e função do corpo, o de atividade e o de participação. Isso faz do AVE a maior causa de incapacidade em países desenvolvidos e em desenvolvimento ${ }^{16,17}$.

No entanto, a incidência das lesões aumenta com a idade e tem seu pico entre a sétima e a oitava décadas de vida quando se somam com as alterações cardiovasculares e metabólicas relacionadas à idade ${ }^{17} \mathrm{e}$ a taxa de incidência anual é de 3,5/1000 para indivíduos entre 55 e 64 anos, e de 9/1000 para indivíduos entre 65 e 74 anos $^{15}$. Depois de um AVE, o paciente passa por fases de recuperação clássicas, que evoluem, do ponto de vista motor, de um período de choque cerebral para o estágio de flacidez muscular e, posteriormente, para um tônus normal, podendo apresentar espasticidade ou ataxia ${ }^{18}$. É comum a queixa de dor muscular e articular e de déficits de equilíbrio e de marcha por esses pacientes. O tratamento fisioterapêutico visa, na maioria das vezes, ao retorno às atividades funcionais, sendo dada pouca importância ao tratamento da postura. No entanto, a postura que se altera em razão do distúrbio tônico pode ser mais um fator na causa das anormalidades de equilíbrio e no movimento funcional desses pacientes ${ }^{2,13}$.

\section{OBJETIVO}

O objetivo deste estudo foi comparar a postura estática e a flexibilidade toracolombar de pacientes hemiplégicos com a de indivíduos de mesma idade, sem história de afecções do sistema nervoso, buscando-se identificar a relação entre alterações tônicas, causadas por AVE, e os encurtamentos musculares, que resultam em disfunções posturais importantes.

\section{CASUÍSTICA E MÉTODOS}

Foi realizado um estudo transversal com convidados a participar indivíduos que apresentaram seqüelas de lesões neurológicas de um AVE e estavam em atendimento fisioterapêutico pelo convênio do Sistema Único de Saúde (SUS), no município de Santos, Estado de São Paulo, e apresentavam marcha independente, com ou sem órtese (muleta/bengala). Um grupo de indivíduos adultos, sem história de lesões neurológicas, foi avaliado como grupo controle. Todos os participantes assinaram o termo de consentimento livre e esclarecido e o desenvolvimento do trabalho foi aprovado pelo Comitê de Ética e Pesquisa (CEP) da Universidade Federal de São Paulo (Unifesp) (CEP n. 1846/06).

\section{Fotometria}

A análise por fotometria foi realizada pelo software para avaliação postural (SAPO), programa de avaliação postural livre, com tutoriais científicos, e por meio da criação de um banco de dados disponibilizado gratuitamente no endereço <http://sapo.incubadora.fapesp. br $>$. Esse programa também permite traçar digitalmente as retas que determinam valores angulares para os pontos de referência correspondentes, posicionados assimetricamente em relação aos dímeros direito e esquerdo do corpo. Os pontos anatômicos foram marcados na pele, pela fixação de bolas de isopor de 2 $\mathrm{mm}$, com auxílio de fita dupla face. Os bilateralmente marcados foram: acrômio, espinha ilíaca ântero-superior, trocânter maior do fêmur, linha articular do joelho, ponto medial da patela, tuberosidade da tíbia, maléolo lateral, medial, ângulo inferior da escápula, processo espinhoso de C7, processo espinhoso de T3, ponto sobre linha média da perna, tendão do calcâneo, espinha ilíaca póstero-superior e ponto entre a cabeça do segundo e terceiro. Os pontos foram marcados em cada vista, sempre na mesma seqüência, totalizando 16 , na vista anterior; 9, na posterior, e 8, nas laterais direita 
e esquerda, respectivamente. Os participantes permaneceram em posição ortostática, em local previamente marcado, à distância de 2,4 m da máquina fotográfica digital (SONY- Cyber Shot, DCS-W30), posicionada paralela ao chão à altura de $1 \mathrm{~m}$, com auxílio de um tripé profissional com prumo. Os voluntários foram fotografados nas vistas anterior, posterior e lateral direita e esquerda, trajando roupa de banho para a visualização dos marcadores. O chão foi marcado para permitir que a posição dos pés fosse mantida em todas as vistas. Na padronização do eixo vertical, utilizou-se um fio de prumo. Pela fotometria, fez-se o cálculo dos ângulos formados pelas linhas entre referências posturais nas vistas, anterior e posterior (acrômios, espinhas ilíacas ântero-superiores e patelas etc.) e a contida no plano horizontal; na vista lateral, calcularam os ângulos de joelho e cotovelo. Descartaram-se os dados das marcações incorretas e aquelas nas quais não foi possível posicionar adequadamente os marcadores, pela presença de obesidade ou resistência do paciente à vestimenta adequada.

\section{Testes de mobilidade da coluna vertebral}

Foram realizados os testes específicos para flexibilidade da coluna, tais como o Índice de Schöber, que avalia a mobilidade lombossacral, e o de Stibor, que verifica toda a coluna vertebral $^{19}$. Pelo Índice de Schöber, o indivíduo ficou em posição ortostática, com os pés juntos; com um lápis dermatográfico, traçouse uma linha unindo as duas espinhas ilíacas póstero-superiores (EIPSs) e, em outra, $10 \mathrm{~cm}$ acima; em seguida, pediu-se ao paciente que realizasse flexão anterior de tronco. O examinador mediu a distância entre as duas linhas demarcadas. É considerado normal o aumento da distância em aproximadamente $5 \mathrm{~cm}$ dos indivíduos que tiveram a mobilidade normal, ou seja, que não apresentaram nenhuma patologia na coluna lombossacral.
O Índice de Stibor foi avaliado com o indivíduo em posição ortostática, com os pés juntos. Com o lápis dermatográfico, traçou-se uma linha unindo as duas EIPSs até encontrar o ponto médio. Em seguida, marcou-se o processo espinhoso da sétima vértebra cervical e, com uma fita métrica, mediu-se a distância entre os dois pontos demarcados. Logo após solicitou-se ao paciente que fizesse flexão anterior do tronco e, nesta posição, o examinador mediu novamente a distância entre os dois pontos. Pela diferença entre esses dois valores mensurados, obtêm-se o Índice de Stibor. Em um indivíduo com mobilidade normal, essa distância aumenta aproximadamente $10 \mathrm{~cm}$. Também foi feita coleta do valor distância mão-chão, uma medida que infere o comprimento da cadeia muscular posterior ${ }^{20}$.

\section{Anólise estatística}

A análise estatística foi realizada com uso do Software Sigma Stat ${ }^{\circledR}$, e pela comparação dos valores de todas as medidas entre o grupo controle e o acometido pelo AVE, por meio do Teste de Soma de Postos de Mann-Whitney, pois nem todos os grupos apresentaram uma distribuição normal. $\mathrm{O}$ valor de $\mathrm{P}$ estabelecido foi 0,05 .

\section{RESULTADOS}

\section{Caracterização da amostra estudada}

Foram avaliados 28 pacientes $(60,82 \pm 11,49$ anos), sendo 18 (64\%) do sexo masculino, e 10 (36\%) do feminino, com histórico de AVE, e 29 indivíduos saudáveis $(51,45 \pm 2,31$ anos), sendo 8 (28\%) do sexo masculino, e 21(72\%), do feminino, que fizeram parte do grupo controle. Todos os pacientes apresentavam mais de seis meses de AVE, casos já considerados crônicos: 14, acometimento no hemisfério cerebral D, e 14, no esquerdo. 


\subsection{Resultados da fotometria}

Depois de todos serem avaliados em um pool, verificou-se que não houve diferença entre os valores dos pacientes com acometimentos nos hemisférios direito e esquerdo $(P>0,05)$. Pela análise da vista anterior, observou-se diferença estatisticamente significativa nos valores superiores de desnível de acrômios, das espinhas ilíacas ântero-superiores e das patelas dos pacientes, na comparação com os indivíduos controle (Tabela 1). Após análise dos dados da vista posterior, verificaram-se os valores superiores de desnível no alinhamento dos ângulos inferiores da escápula, das espinhas ilíacas póstero-superiores e das linhas poplíteas dos indivíduos acometidos pelo AVE, comparados aos do grupo controle (Tabela 2). Pela análise da vista lateral, não foi observada diferença estatisticamente significativa entre os valores de ângulos de joelho e cotovelo dos pacientes e dos indivíduos do grupo controle (Tabela 3).

\section{Resultados dos índices de mobilida- de vertebral e comprimento de cadeia posterior}

Houve diferenças estatisticamente significativas dos valores dos Índices de Schöber e Stibor entre os pacientes e os indivíduos do grupo controle, sendo os primeiros inferiores. Em relação à distância mão-chão, o grupo acometido apresentou valores maiores que o controle (Tabela 4).

\section{DISCUSSÃO}

Os propósitos estabelecidos no planejamento desta pesquisa foram atingidos, com a identificação das assimetrias posturais em pacientes hemiplégicos, por seqüela motora de acidente vascular encefálico. Nem todas as possibilidades de avaliação permitidas pelo software foram utilizadas, pois muitos pacientes resistiam ao uso de pouca vestimenta necessária para a avaliação, e alguns pontos
Tabela l: Média, erro padrão da média (†EPM) valores de P para comparação da variável ângulo do vértice formado por linhas entre referências posturais da vista anterior (acrômios, espinhas ilíacas ântero-superiores e patelas) e o plano horizontal, entre os pacientes e o grupo controle

\begin{tabular}{ccccc}
\hline \multirow{2}{*}{ Grupo } & $\begin{array}{c}\text { Referência } \\
\text { óssea }\end{array}$ & Acrômios & EIAS & Patelas \\
\hline \multirow{4}{*}{ Controle } & Média & 1,45 & 0,66 & 0,84 \\
& $\mathrm{~N}$ & 29 & 22 & 29 \\
& \pm EPM & 0,22 & 0,11 & 0,12 \\
& Média & 3,3 & 2,15 & 2,17 \\
Pacientes & $\mathrm{N}$ & 28 & 21 & 28 \\
& \pm EPM & 0,38 & 0,29 & 0,27 \\
Valor P & & $<0,0001$ & $<0,0001$ & $<0,0001$ \\
\hline
\end{tabular}

EIAS: Espinha Ilíaca Ântero-superior.

Tabela 2: Média, erro padrão da média ( \pm EPM) e valores de P da variável ângulo do vértice, formado por linhas entre referências posturais da vista posterior (ângulo inferior das escápulas, espinhas ilíacas póstero-superiores e linhas poplíteas) e o plano horizontal

\begin{tabular}{ccccc}
\hline Grupo & $\begin{array}{c}\text { Referência } \\
\text { óssea }\end{array}$ & $\begin{array}{c}\text { A. I. } \\
\text { Escápula }\end{array}$ & EIPIS & $\begin{array}{c}\text { Linha } \\
\text { poplítea }\end{array}$ \\
\hline \multirow{4}{*}{ Controle } & Média & 1,40 & 1,27 & 0,88 \\
& $\mathrm{~N}$ & 29 & 29 & 29 \\
& \pm EPM & 0,17 & 0,19 & 0,15 \\
& Média & 4,99 & 3,08 & 1,92 \\
Pacientes & $\mathrm{N}$ & 28 & 28 & 28 \\
& \pm EPM & 0,87 & 0,40 & 0,26 \\
Valor P & & $<0,0001$ & $<0,0001$ & $<0,0001$ \\
\hline
\end{tabular}

EIAS: Espinha Ilíaca Póstero-superior; AIEscápula: Ângulo inferior da escápula.

Tabela 3: Média e erro padrão da média ( \pm EPM) dos ângulos de joelho e cotovelo dos grupos controle e acometido, para os dois hemicorpos

\begin{tabular}{ccccc}
\hline \multicolumn{2}{c}{ Grupo } & Joelho & Cotovelo \\
\hline \multirow{4}{*}{ Hermicorpo } & \multirow{2}{*}{ Controle } & Média & 177,85 & 150,38 \\
D & & \pm EPM & 1,15 & 1,78 \\
& \multirow{2}{*}{ Pacientes } & Média & 171,74 & 155,99 \\
& & \pm EPM & 2,51 & 2,25 \\
Hermicorpo & \multirow{2}{*}{ Controle } & Média & 174,22 & 149,67 \\
E & & \pm EPM & 2,04 & 1,61 \\
& \multirow{2}{*}{ Pacientes } & Média & 174,19 & 149,97 \\
& & \pm EPM & 2,27 & 2,51 \\
\hline
\end{tabular}


Tabela 4: Médias e erro padrão das médias ( \pm EPM) dos valores dos Índices de Schöber e Stibor e da Distância Mão-chão para os pacientes avaliados e o grupo controle

\begin{tabular}{lcccc}
\hline \multirow{2}{*}{ Grupo } & & $\begin{array}{c}\text { Índice } \\
\text { de Shober }\end{array}$ & $\begin{array}{c}\text { Índice } \\
\text { de Stibor }\end{array}$ & $\begin{array}{c}\text { Distância } \\
\text { mão-chão }\end{array}$ \\
\cline { 2 - 5 } Controle & Média & 14,31 & 7,31 & 9,55 \\
& $\mathrm{~N}$ & 29 & 29 & 29 \\
& \pm EPM & 0,18 & 0,41 & 1,65 \\
& Média & 14,31 & 6,46 & 24,08 \\
Pacientes & $\mathrm{N}$ & 27 & 27 & 28 \\
& EEPM & 0,2 & 0,34 & 2,28 \\
Valor P & & 0,0300 & 0,0472 & $<0,0001$ \\
\hline
\end{tabular}

não puderam fazer parte da análise estatística para todos eles. No entanto, foi possível observar que as assimetrias posturais apresentadas pelos pacientes são pertinentes e devem ser ressaltadas nos objetivos de tratamento físico desses indivíduos.

O método proposto para quantificação das assimetrias posturais pela fotometria é satisfatoriamente confiável para a maioria das medidas analisadas ${ }^{21}$ e os resultados dos pacientes estudados indicam que indivíduos acometidos por um acidente vascular encefálico apresentam grande assimetria postural, representada pelos maiores desníveis entre os segmentos, cintura escapular e pélvica e membros inferiores. No entanto, os valores da distância mão-chão indicam um encurtamento da cadeia muscular posterior dos pacientes e os Índices de Schöber e Stibor mostram a redução da mobilidade vertebral, o que pode levar à perda da mobilidade funcional da coluna e a dores articulares e musculares. Todas essas alterações identificadas, em conjunto, causam, a médio e a longo prazos, o desenvolvimento de comprometimentos secundários como contraturas, deformidades articulares e prejuízos na execução das atividades de vida diária, alterações clássicas observadas pelos fisioterapeutas há muito tempo ${ }^{13}$.

Muitos fatores podem ser considerados os causadores dessa assimetria nos pacientes hemiplégicos. As alterações tônicas, comuns após lesões neurológicas, são consideradas comprometimentos primários do controle do movi- mento ${ }^{2}$. Outro fator importante para a manutenção da postura é a propriocepção. Segundo $\mathrm{O}^{\prime}$ Sullivan ${ }^{13}$, Moura ${ }^{22}$ e Lent $^{23}$, a diminuição da propriocepção, pela redução da aferência dos receptores de cápsulas, ligamentos, músculos e tendões, é uma conseqüência comum dos distúrbios do sistema nervoso central, o que prejudica a correção postural e a manutenção da consciência corporal. A acomodação do SNC à nova postura e à condição motora poderia ser também ressaltada. Segundo Watkins ${ }^{24}$, quando um estímulo sensorial (como o proprioceptivo) é aplicado, a resposta inicial dos receptores é a transmissão de uma taxa de impulso acima dos valores de normalidade, que vai diminuindo progressivamente até finalmente não responder a nada, esperando-se, assim, uma acomodação do SNC ao input mecanorreceptor. Então, todos os receptores se adaptam, parcial ou completamente, ao estímulo recebido. É provável que haja acomodação do SNC à redução de atividade dos indivíduos após afecção neurológica, o que seria apenas o início de uma grande modificação no alinhamento postural antigravitacional.

Esses pacientes demandam, durante o tratamento fisioterapêutico, especial atenção na avaliação postural, pois o alinhamento dos segmentos corporais é um dos fatores biomecânicos principais para um controle motor adequado $^{25}$. Seria preciso um tratamento estático específico para alterar o alinhamento postural dos pacientes, por meio de input sensorial normal, e, dessa forma, reestruturar as respostas plásticas do SNC, o que proporcionará uma base neurofisiológica mais adequada ao controle da postura ${ }^{26}$. É reconhecido que as afecções neurológicas geram disfunções motoras, que podem permanecer para déficits residuais e posturais importantes, o que prejudica as capacidades físicas e as interações sociais do indivíduo ${ }^{16}$. Este estudo contribuiu com essa afirmativa no que se refere à comprovação dos dados quantitativos das assimetrias na postura estática, com a utilização de um instrumento gratuito disponível pela internet. 


\section{CONCLUSÃO}

Os resultados desta pesquisa indicam que é preciso dar mais atenção à avaliação postural de pacientes neurológicos que apresentam desvios posturais importantes e que a reeducação estática como terapia deve ser mais considerada nesses casos. Deve-se levar em consideração também a possibilidade da aplicação dos princípios de reequilíbrio tônico por meio da manipulação das cadeias musculares, o que proporcionaria um tratamento mais eficaz e integral ao paciente que demonstra comprometimento global do sistema músculo-esquelético.

\section{APOIO FINANCEIRO: CNPq}

\section{REFERÊNCIAS}

1. Kisner C, Colby LAA. Exercícios terapêuticos. São Paulo: Manole, 2005.

2. Shumway-Cook A, Woollacott MH. Controle motor: teoria e aplicações práticas. $2^{\mathrm{a}}$ ed. São Paulo: Manole, 2003.

3. Bienfait M. Bases elementares técnicas de terapia manual e osteopatia. São Paulo: Summus, 1997.

4. Bienfait M. Estudo e tratamento de esqueleto fibroso: fáscias e pompages. São Paulo: Summus, 1999.

5. Ferrario VF, Sforza C, Tartaglia G, Barbini E, Michielon G. New television technique for natural head and body posture analysis. The J Craniomand Practice. 1995;13(4):24-55.

6. Watson AWS, Macdonncha C. A reliable technique for the assessment of posture: assessment criteria for aspects of posture. J Sports Med Phys Fitness. 2000;40(3):260-70

7. Kendal FP, Mccreary EK, Provance PG. Músculos: provas e funções. São Paulo: Manole, 1997.

8. Saxton JB. Postural alignment in standing: a repeatability study. Australian Physioltherapy. 1993; 39(1):25-9.

9. Freeres M, Mairlot MB. Maítres et clés de la posture. Paris: Frison-Roche, 1997.
10. Horak FB, Macpherson JM. Postural orientation and equilibrium. In: Rowell LB, Shepherd JT, editors. Handbook of physiology. Oxford University Press: New York, 1996.

11. Amado-João MS. Métodos de avaliação clínica e funcional em fisioterapia. Rio de Janeiro: Guanabara Koogan, 2006.

12. Doretto D. Fisiopatologia clínica do sistema nervoso: fundamentos da semiologia. Rio de Janeiro: Atheneu, 1998. 465 p.

13. O' Sullivan SB. Acidente vascular encefálico. In: $\mathrm{O}^{\prime}$ Sullivan SB, Schmitz TJ. Fisioterapia avaliação e tratamento. $4^{\text {a }}$ ed. São Paulo: Manole, 2004.

14. Smith LK, Weiss EL, Lehmkuhl LD. Cinesiologia clínica de Brunnstrom. São Paulo: Manole, 1997.

15. Organização Mundial de Saúde. Promovendo qualidade de vida após um acidente vascular cerebral: um guia para fisioterapeutas e profissionais de atenção primária à saúde. Porto Alegre: Artmed, 2003.

16. Teixeira-Salmela LF, Faria CDCM, Guimarães CQ, Goulart F, Parreira VF, Inácio EP, Alcântara TO. Treinamento físico e destreinamento em hemiplégicos crônicos: impacto na qualidade de vida. Rev Bras Fisioter. 2005;9(3):347-53

17. Zétola VHF, Nóvak EM, Camargo CHF, Carraro Júnior H, Coral P, Muzzio JA, et al. Acidente vascular cerebral em pacientes jovens. Arquivo de Neuropsiquiatria. 2001;59(3-B):740-45.

18. Cohen H. Neurociência para fisioterapeutas: incluindo correlações clínicas. São Paulo: Manole, 2001.

19. Marques AP. Manual de goniometria, São Paulo: Manole, 2003.

20. Santos A. Diagnóstico clínico postural: um guia prático. São Paulo: Summus, 2001.

21. Lunes DH, Castro FA, Moura IC, Salgado HS, Oliveira AS, Bevilaqua-Grossi, D. Confiabilidade intra e interexaminadores e repetibilidade da avaliação postural pela fotogrametria. Rev. Bras de Fisioter. 2005;9(3):327-34.

22. Moura RN, Santos FC, Driemeier M, Santos LM dos, Ramos LR. Quedas em idosos: fatores de risco associados. Gerontologia. 1999;7(2):15 -21.

23. Lent R. Cem milhões de neurônios: conceitos fundamentais de neurociência. São Paulo: Atheneu; 2001. 
24. Watkins C. Mechanical and neurophysiological changes in spastic muscles: serial casting in spastic equinovarus following traumatic brain injury.

Physiotherapy. 1999;85(11):603-9.

25. Gurfinkel VS, Cordo PJ. The scientific legacy of Nikolai Bernstein. In: Latash M, editors. Progress in Motor Control. v.1: Bernstein's tradition in movement studies. Champaign, II. Human Kinetics, 1998.
26. Seil FJ. Neural plasticity and regeneration. New York: Elsevier; 2000. 